\title{
Ludicidade e método ativo na educação alimentar e nutricional do escolar
}

\author{
Playfulness and active method in food and nutricional \\ education of schoolchildren
}

\author{
Adriene Carvalho da Conceição', Erica Costa da Costa', Leandro Orlando Sousa da Silva², \\ Elen Dayane Albuquerque Cardoso', Bárbara Valéria de Souza Santos', \\ Elaine do Socorro Brabo Duarte', Xaene Maria Fernandes Duarte de Mendonça³, \\ Réia Silvia Lemos da Costa e Silva Gomes ${ }^{4}$ \\ ' Faculdade de Nutrição, Universidade Federal do Pará, Belém (PA), Brasil. \\ ${ }^{2}$ Instituto de Ciências Exatas e Naturais, Universidade Federal do Pará, Belém (PA), Brasil. \\ ${ }^{3}$ Instituto de Ciências Biológicas, Universidade Federal do Pará, Belém (PA), Brasil. \\ ${ }^{4}$ Faculdade de Nutrição, Instituto de Ciências da Saúde, Universidade Federal do Pará, Belém (PA), Brasil.
}

Recebido: Ago. 15, 2018 Aceito: Jun. 24, 2019

\section{COMO CITAR ESTE ARTIGO}

Conceição AC, Costa EC, Silva LOS et al. Ludicidade e método ativo na educação alimentar e nutricional do escolar. Interdisciplinary Journal of Health Education. 2019 Jan-Dez:4(1-2):34-41. https://doi.org/10.4322/ijhe.2018.009

\section{CORRESPONDÊNCIA}

Adriene Carvalho da Conceição Rua Augusto Corrêa, 1, Guamá, CEP 66075-900, Campus IV, Saúde, Belém (PA), Brasil

adrienecarvalho18@gmail.com

\section{FONTE DE FINANCIAMENTO} Programa Eixo Transversal 2017/2018, financiado parcialmente pela Pró-Reitoria de Extensão, da Universidade Federal do Pará.

\section{CONFLITO DE INTERESSE}

Os autores declararam não

haver conflitos de interesse.

Estudo realizado em duas escolas da rede pública de ensino municipal em Belém-PA; cujas atividades fizeram parte do projeto "Cultivando Hortas Escolares como Eixo Hortas Escolares como Eixo
Gerador de Segurança Alimentar Nutricional e Sustentabilidade

\section{RESUMO}

Introdução: A escola pode contribuir para o desenvolvimento de hábitos saudáveis por meio de atividades capazes de envolver e construir conhecimento sobre a alimentação e nutrição. Objetivo: O estudo visa avaliar três ações educativas realizadas por meio de metodologias ativas com escolares do ensino fundamental, abordando alimentos regionais, promoção da alimentação saudável e sustentabilidade. Método: Foi realizado um estudo transversal, quantiqualitativo, analítico-descritivo a partir das atividades educativas teatro de fantoches, roda de conversa e oficina culinária, com avaliação de um teste de conhecimento e de aceitabilidade de um lanche com ingredientes regionais. Resultados: A amostra consistiu de 49 estudantes, entre 7 e 10 anos. No conhecimento assimilado não se observou diferença estatística entre os alunos das duas escolas; a aceitabilidade do lanche regional na Escola $A$ foi ótima $(95,8 \%)$ e boa na Escola B $(84,0 \%)$, mas não se observou diferença estatística na aceitabilidade geral. Conclusões: As metodologias ativas aplicadas no desenvolvimento de EAN demonstraram ser eficazes no processo de aquisição do conhecimento a respeito do que são alimentos regionais, alimentação saudável e valorização da cultura alimentar local.

PALAVRAS-ChaVE: Alimentação escolar. Alimentação saudável. Educação.

\section{ABSTRACT}

Introduction: The school can contribute to the development of healthy habits through activities that can engage and build knowledge about food and nutrition. Objective: The study aims to evaluate three educational actions carried out through active methodologies with primary school students, addressing regional foods, promoting healthy eating and sustainability. Method: A cross-sectional, quantitative, analytical-descriptive study was carried out from the puppet theater, conversation wheel and culinary workshop, with a test of knowledge and acceptability of a snack with regional ingredients. Results: The sample consisted of 49 students, between 7 and 10 years old. In the assimilated knowledge there was no statistical difference between the students of the two schools; in the acceptance test of the regional snack in School A was good (95.8\%) and good in School B (84.0\%), but no statistical difference was observed in overall acceptability. Conclusions: The active methodologies applied in the development of EANs have been shown to be effective in the process of acquiring knowledge about what are regional foods, healthy food and valorization of the local food culture.

KEYWORDS: School feeding. Healthy diet. Education. 


\section{Introdução}

A escola é um espaço de promoção da saúde porque trabalha a formação do cidadão, a sua autonomia, o exercício dos direitos e deveres, o controle das condições de saúde e a qualidade de vida na obtenção de comportamentos e atitudes consideradas saudáveis ${ }^{1}$.

Para a Sociedade Brasileira de Pediatria² ${ }^{2}$ o período escolar, na faixa etária entre 7 a 10 anos, tem grande importância no desenvolvimento físico e intelectual, portanto, a escola é um ambiente que pode contribuir para a formação de hábitos saudáveis por meio de atividades capazes de envolver os alunos e construir conhecimento, principalmente no que diz respeito à alimentação e nutrição e os cuidados referentes à saúde.

Para que haja uma alimentação adequada é necessário estar atento às escolhas alimentares e a valores como cuidado ambiental, apropriação da cultura e costumes locais. Como o Brasil é um país de ampla diversidade cultural e alimentar é possível destacar os alimentos regionais como elementos componentes do "prato" do brasileiro, por sua representatividade, valorização, compartilhamento de tradições e valor nutritivo, que influenciam positivamente na saúde e bem-estar da população, diferentemente dos alimentos industrializados ${ }^{3}$.

Os alimentos regionais são aqueles típicos de determinada região, que possuem alto valor nutritivo e que no período de safra têm-se fácil acesso e custo reduzido, o que os tornam excelentes opções de consumo para o combate à insegurança alimentar de crianças, que estão consumindo em demasia produtos ultraprocessados, em detrimento dos alimentos típicos das regiões a que pertencem ${ }^{4}$. No entanto, a cultura alimentar tradicional encontra-se cada vez mais prejudicada pelo consumo indiscriminado de alimentos ultraprocessados. Essa prática alimentar associada a outros hábitos de vida inadequados aumentam as chances de alterações no estado nutricional e de aquisição de Doenças e Agravos Não Transmissíveis (DANT), desde a infância até a fase adulta 5 .

O público infantil tem adquirido hábitos alimentares prejudiciais, por esse motivo são necessárias estratégias capazes de incentivar a alimentação saudável, com a inclusão de alimentos da cultura regional, mediante a difusão do conhecimento sobre a relevância dos mesmos, promovendo assim a saúde com a utilização de atividades de Educação Alimentar e Nutricional $(E A N)^{6}$, que podem ser realizadas por meio das metodologias ativas de educação, como forma de otimizar o aprendizado, uma vez que são consideradas excelentes estratégias de ensino-aprendizagem ${ }^{7,8}$. Com a utilização dessa estratégia de aprendizagem o professor assume o papel de facilitador, dando espaço para o aluno buscar o conhecimento de forma autônoma, tornando o ambiente de educação ativo, dinâmico e construtivo, estimulando o senso crítico e interferindo positivamente no desempenho escolar. Dentre os métodos que se consideram metodologias ativas, caracterizadas por serem "construtivistas, colaborativos, interdisciplinares, contextualizados, reflexivos, críticos, investigativos, humanistas, motivadores e desafiadores"7; são exemplos o Problem Based Learnig (PBL), Roda de conversa, Team Based Learning (TBL), Problematização, Aprendizagem Baseada em Projetos ou Aprendizagem por Projetos (APP).

O estudo visa, então, avaliar três ações educativas realizadas por meio de metodologias ativas com escolares do ensino fundamental de duas escolas públicas do Pará, abordando alimentos regionais e promoção da alimentação saudável.

\section{Material e métodos}

Trata-se de um estudo transversal, quantiqualitativo, analítico-descritivo, realizado em duas escolas da rede pública municipal de ensino, no estado do Pará, desenvolvido no período de fevereiro a abril de 2018. O projeto de pesquisa foi aprovado pelo 
Comitê de Ética em Pesquisa da Universidade Federal do Pará (UFPA), sob o CAAE 56171415.1.0000.0018.

O público-alvo foi composto de estudantes do $3^{\circ}$ ano do Ensino Fundamental, com amostragem de $n=49$, sendo na escola a $n=24$ e escola $b n=25$, de ambos os sexos, com faixa etária dos 7 aos 10 anos. Os alunos foram selecionados por conveniência e os critérios de inclusão no estudo foram a concordância em participar voluntariamente, assinatura do termo de consentimento livre esclarecido (TCLE) pelos pais e ter presença em todas as atividades, já os critérios de exclusão foram a recusa em participar, a não autorização dos pais por meio da assinatura do TCLE e ter faltado em um dos três encontros.

As metodologias ativas de educação foram utilizadas para realizar a EAN dos escolares, três momentos em cada escola. No primeiro momento aplicou-se o roteiro de um teatro de fantoches, com o conteúdo de alimentos regionais e alimentação saudável e sustentável, com o intuito de incentivar a apropriação da cultura alimentar regional, promover saúde e consciência ambiental. Os recursos didáticos utilizados foram um cenário com três fantoches, quatro palitoches de alimentos regionais e um folder educativo, todos elaborados pelos acadêmicos autores da pesquisa. Ao final desta atividade os alunos leram o folder em conjunto e estabeleceu-se um diálogo, com exposição de dúvidas e comentários sobre o que eles compreenderam a respeito do conteúdo da atividade. As impressões sobre a aceitação e envolvimento da turma foram registradas em forma de anotações, por meio de observação direta feita por um avaliador (acadêmico e autor do projeto).

No segundo momento trabalhou-se uma roda de conversa, que teve como cenário uma minifeira, com a exposição de alimentos regionais e uso de banner com a mesma temática, como recurso pedagógico para o diálogo, sugerindo assim a visita à uma feira para conhecer a utilização dos alimentos regionais na culinária. Da mesma forma que ocorreu no teatro de fantoches, a efetividade da atividade foi avaliada por observação direta. Terminado esse momento, foi aplicado um teste de conhecimento, elaborado pelos autores da pesquisa, sobre o conteúdo trabalhado nessa atividade e na anterior, onde na ficha do teste os alunos também preencheram informações de idade, sexo e escola, como descrito nos anexos (Tabela 1).

Tabela 1. Características gerais dos estudantes das Escolas A e B e Avaliação do Conhecimento sobre alimentos regionais. Belém/PA, 2018.

\begin{tabular}{|c|c|c|c|c|c|c|c|}
\hline \multirow{2}{*}{ Idade } & \multicolumn{2}{|c|}{ Escola A } & \multicolumn{2}{|c|}{ Escola B } & \multicolumn{2}{|c|}{ Geral } & \multirow{2}{*}{$\begin{array}{l}A \times B \\
\text { p-valor }\end{array}$} \\
\hline & $\mathbf{N}$ & $\%$ & $\mathbf{N}$ & $\%$ & $\mathbf{n}$ & $\%$ & \\
\hline 7 anos & 0 & 0 & 1 & 4.0 & 1 & 2.0 & \multirow{4}{*}{0.1517} \\
\hline 8 anos & 21 & 87.5 & 16 & 64.0 & 37 & 75.5 & \\
\hline 9 anos & 3 & 12.5 & 1 & 4.0 & 4 & 8.2 & \\
\hline 10 anos & 0 & 0 & 7 & 28.0 & 7 & 14.3 & \\
\hline \multicolumn{8}{|l|}{ Sexo } \\
\hline Masculino & 12 & 50 & 12 & 48.0 & 24 & 49.0 & \multirow{2}{*}{0.8841} \\
\hline Feminino & 12 & 50 & 13 & 52.0 & 25 & 51.0 & \\
\hline \multicolumn{8}{|l|}{ Avaliação do Conhecimento } \\
\hline Pergunta 1 - Alimentação Saudável. & 24 & 100.0 & 25 & 100.0 & 49 & 100.0 & 1.0000 \\
\hline Pergunta 2 - Consumo Alimentar. & 23 & 95.8 & 24 & 96.0 & 47 & 95.9 & 0.4885 \\
\hline Pergunta 3 - Consumo Alimentar. & 3 & 12.5 & 0 & 0.0 & 3 & 6.1 & 0.1999 \\
\hline Pergunta 4 - Alimentação Regional. & 23 & 95.8 & 24 & 96.0 & 47 & 95.9 & 0.4885 \\
\hline Pergunta 5 - Consumo de alimentos regionais. & 24 & 100.0 & 25 & 100.0 & 49 & 100.0 & 1.0000 \\
\hline Pergunta 6 - Exemplos de alimentos regionais. & 24 & 100.0 & 25 & 100.0 & 49 & 100.0 & 1.0000 \\
\hline
\end{tabular}

Nota: A estatística inferencial - Teste do Qui-quadrado de independência. 
No terceiro momento os escolares participaram de uma Oficina Culinária, na qual produziram um lanche saudável com ingredientes regionais; nesta etapa da atividade, um avaliador registrou o interesse e participação dos alunos utilizando como instrumento de avaliação a observação direta. Os participantes consumiram o lanche e responderam um teste de aceitabilidade, que continha uma escala hedônica facial mista de 5 pontos, contendo desenhos de expressões faciais, recomendado pelo FNDE ${ }^{9}$, que estabelece que para se considerar adequada a aceitação de um lanche é necessário alcançar o índice mínimo de $85 \%$ de aceitação, constituído pela somatória das opções "gostei" e "adorei"10,11. A ficha apresentava expressões, identificadas com números e palavras correspondentes às expressões faciais: 5- Adorei, 4- Gostei, 3-Indiferente, 2- Não gostei, 1- Detestei; na qual as crianças deveriam marcar a opção que correspondesse a sua aceitação.

A variáveis categóricas obtidas nos três encontros nas duas escolas foram coletados por meio de observação direta, com anotações sobre o comportamento e indicadores de compreensão da temática abordada e por preenchimento do teste de conhecimento, onde registrou-se informações de sexo, idade e escola.

Os dados coletados foram tabulados no programa dEASYgner versão $2.0 \mathrm{e}$ analisados no programa BioEstat versão $5.3^{12}$. Aplicaram-se os métodos estatísticos descritivos e inferenciais na análise do Teste de conhecimento e do Teste de Aceitação do Lanche; as variáveis qualitativas (idade, sexo e conhecimento) apresentaram-se por meio de distribuições de frequências absolutas e relativas e, as variáveis quantitativas (aceitabilidade) foram apresentadas por medidas de tendência central e de variação.

\section{Resultados}

A amostra da pesquisa foi constituída por 49 estudantes do $3^{\circ}$ ano do ensino fundamental, 24 (49\%) da Escola A e 25 (51\%) da Escola B; 25 do sexo feminino e 24 do sexo masculino, com idade média de 8,5 anos.

$\mathrm{Na}$ atividade do Teatro de Fantoches foi trabalhado o tema "Reconhecimento e apropriação da cultura alimentar regional e práticas alimentares saudáveis". Os alunos das duas escolas demonstraram estar atentos aos acontecimentos da encenação ao esboçarem reações de suspense, surpresa e alegria ou interagirem com os personagens; após a encenação iniciava-se uma conversa acerca das informações contidas no folder educativo ilustrado e dos acontecimentos da encenação. Os alunos da Escola A $(n=24)$ participaram ativamente com questionamentos sobre alimentos regionais, elaboração de receitas com o uso dos alimentos regionais, relatos de idas à feiras da localidade onde residem, das preferências de consumo dos alimentos regionais, dentre outras. Os alunos da Escola $B(n=25)$ foram menos participativos, pois somente se manifestaram quando solicitados a fazer leitura do folder ou a responder sobre o assunto abordado; relataram suas preferências com relação aos alimentos típicos da região Norte, frequência de consumo destes alimentos e episódios de idas a feiras de suas localidades, quando demonstraram interesse e participaram de forma ativa.

$\mathrm{Na}$ atividade Roda de Conversa com o tema "Consumo de alimentos regionais na promoção da alimentação saudável e da segurança alimentar e nutricional", pouca diferença foi observada na participação entre os alunos das duas escolas; notou-se grande interesse dos escolares, pois a cada pergunta formulada os participantes demonstraram grande vontade em responder e fazer novas perguntas. Na vivência do cenário de 'feirinha', os alunos conheceram alguns alimentos não vistos ou não consumidos ou reconheceram alimentos típicos da região Norte, alguns já consumidos.

Coletaram-se características referentes aos dados de idade, sexo e nível de conhecimento assimilado pelos estudantes das escolas A e B verificado por meio do Teste de conhecimento aplicado após o Teatro de Fantoches e a Roda de Conversa. O teste foi aplicado após a roda de conversa e os dados das duas escolas não apresentaram diferença estatística significante em todas as respostas $(p>0,005)$, indicando que os estudantes compreenderam que a alimentação saudável é importante 
para o bom funcionamento do organismo; quais alimentos compõem uma alimentação saudável (feijão, arroz, salada, peixe e manga) e quais alimentos não são adequados ao consumo (refrigerante e biscoito recheado); por qual motivo é aconselhável o consumo dos alimentos típicos da região, reconhecendo os alimentos típicos: cupuaçu, pupunha, manga, jambu, chicória, bacuri, castanha do Pará (Tabela 1).

Nota-se que não houve significância estatísticas entre as variáveis idade e sexo; na verificação do nível de acertos e erros dos escolares no teste de fixação $100 \%$ dos alunos responderam acertadamente às perguntas 1, 5 e 6 que abordavam, respectivamente, a importância da alimentação saudável para o bom funcionamento do organismo, as vantagens do consumo dos alimentos regionais, o aspecto nutricional e econômico, tendo como exemplos alimentos típicos da região Norte, como cupuaçu, jambu, pupunha; a pergunta 3, que tratava sobre o consumo de ultraprocessados, foi a que apresentou maior percentual de erros, pois 6,1\% dos estudantes, todos da Escola A, responderam erroneamente. Comparando-se as duas escolas, no geral, não há real diferença estatística de conhecimentos assimilados ( $p>0,05)$ (Tabela 1).

$\mathrm{Na}$ atividade da Oficina Culinária de lanche saudável com alimentos regionais o tema trabalhado foi o "Desenvolvimento de habilidades culinárias e valorização de alimentos regionais". Os escolares elaboraram duas receitas: um cookie e um suco de fruta regional; a atividade gerou grande satisfação e os alunos assimilaram de forma satisfatória a maioria das informações repassadas desde a primeira atividade realizada, uma vez que souberam reconhecer os alimentos regionais utilizados na receita - a castanha do Pará e o cupuaçu - e souberam informar por que é aconselhável o consumo dos alimentos regionais. Após o consumo do lanche saudável foi aplicado o Teste de Aceitação do lanche à base de alimentos típicos da região.

Os dados do Teste de Aceitação do Lanche variaram em critérios de pontuação de 0 a 100 pontos. Observa-se na Tabela 2 que houve ótima aceitabilidade do lanche ofertado na Escola A, com mediana de 100 pontos, e boa aceitabilidade na Escola B, com mediana de 75 pontos, expressando significativa diferença estatística na aceitabilidade entre as escolas $(p<0,0035)$.

Tabela 2. Aceitabilidade do lanche ofertado aos estudantes das Escolas A e B, Belém/PA, 2018.

\begin{tabular}{|c|c|c|c|}
\hline & Escola A & Escola B & Geral \\
\hline & $(n=21)$ & $(n=25)$ & $(n=46)$ \\
\hline Mínimo & 75.0 & 50.0 & 50 \\
\hline Máximo & 100.0 & 100.0 & 100 \\
\hline Mediana & 100.0 & 75.0 & 100 \\
\hline Primeiro Quartil & 100.0 & 75.0 & 75 \\
\hline Terceiro Quartil & 100.0 & 100.0 & 100 \\
\hline Média Aritmética & 95.8 & 84.0 & 89.4 \\
\hline Desvio Padrão & 8.2 & 13.3 & 12.6 \\
\hline Coeficiente de Variação & $8.59 \%$ & $15.80 \%$ & $14.30 \%$ \\
\hline
\end{tabular}

Nota: Estatística inferencial - Teste U de Mann-Whitney, p-valor = 0,0035.

No teste foram realizados três questionamentos: 1) O que mais gostou? 2) O que menos gostou? 3) Consumiria este lanche se fizesse parte da merenda escolar? $\mathrm{Na}$ Tabela 3 observa-se a preferência por componentes do lanche ofertado e, se consumiriam o lanche caso fosse ofertado na merenda escolar, onde $98 \%$ o consumiriam na merenda escolar; não apresentando diferença estatística no consumo entre as escolas A e B. O cookie foi a preparação preferida e o suco a menos apreciada em ambas as escolas. 
Tabela 3. Avaliação dos questionamentos investigados no teste de aceitabilidade do lanche ofertado aos estudantes das Escolas A e B, localizadas na região metropolitana de Belém/PA, 2018.

\begin{tabular}{|c|c|c|c|c|c|c|c|}
\hline & \multicolumn{2}{|c|}{ Escola A } & \multicolumn{2}{|c|}{ Escola B } & \multicolumn{2}{|c|}{ Geral } & \multirow{2}{*}{ p-valor } \\
\hline & $\mathbf{n}$ & $\%$ & $\mathbf{n}$ & $\%$ & $\mathbf{n}$ & $\%$ & \\
\hline Mais gostou & & & & & & & $0.0113^{*}$ \\
\hline Cookie & 3 & 12.5 & 9 & 36.0 & 12 & 24.5 & \\
\hline Suco & 0 & 0.0 & 5 & 20.0 & 5 & 10.2 & \\
\hline $\begin{array}{c}\text { Gotas } \\
\text { chocolate }\end{array}$ & 2 & 8.3 & 1 & 4.0 & 3 & 6.1 & \\
\hline $\begin{array}{l}\text { Cookie e } \\
\text { suco }\end{array}$ & 19 & 79.2 & 10 & 40.0 & 29 & 59.2 & \\
\hline Menos gostou & & & & & & & $0.0044^{*}$ \\
\hline Nada & 22 & 91.7 & 12 & 48.0 & 34 & 69.4 & \\
\hline Cookie & 1 & 4.2 & 5 & 20.0 & 6 & 12.2 & \\
\hline Suco & 1 & 4.2 & 8 & 32.0 & 9 & 18.4 & \\
\hline $\begin{array}{l}\text { Consumiria na } \\
\text { escola }\end{array}$ & & & & & & & 0.9998 \\
\hline Sim & 24 & 100.0 & 24 & 96.0 & 48 & 98.0 & \\
\hline Não & 0 & 0.0 & 1 & 4.0 & 1 & 2.0 & \\
\hline
\end{tabular}

*Real diferença estatística na aceitabilidade do lanche pelos alunos. $\mathrm{p}$-valor $=<0,05$.

\section{Discussão}

A amostra de escolares da pesquisa não apresentou diferença estatística com relação ao sexo com relativa uniformidade na amostra. O predomínio do sexo feminino (51\%) foi observado por Filgueiras et al. ${ }^{13}$ (51\%) e Silva et al. ${ }^{14}(63,64 \%)$. A idade dos participantes $(p$-valor $=0.1517)$ não interferiu de forma relevante na aprendizagem dos mesmos.

A ampla participação dos estudantes na atividade teatro de fantoches, demonstrada por meio de atenção, na interação com os personagens, na discussão acerca do tema abordado, foi igualmente relatada por Luchetti et al. ${ }^{15}$ em estudo com crianças de faixa-etária similar ( 8 a 9 anos) e que consideraram ser excelente estratégia de ensino em saúde, uma vez que são capazes de prender a atenção e estimular a participação; Aringhieri e Silva ${ }^{16}$ observaram assimilação de conteúdos complexos para a idade.

A Roda de Conversa é uma metodologia ativa de ensino por possibilitar ao aluno expressar suas opiniões e dúvidas, a comunicar e formular justificativas que embasem suas opiniões, a interagir com seus pares e com o professor, tornando-o mais crítico e participativo ${ }^{17}$ num aprendizado mais significativo. No estudo de Dias et al. ${ }^{18}$ foi verificado que a metodologia possibilita a disseminação de conhecimento, esclarecimento de dúvidas e a aproximação entre os atores da conversa, tal como se constatou no presente estudo, onde tanto os alunos das Escola A quanto da Escola B manifestaram ampla participação e interesse nas atividades, com questionamentos, expressão de opiniões, relatos de experiências, etc.

Na presente pesquisa foi possível demonstrar aos escolares, com as oficinas culinárias e a degustação das preparações elaboradas, como os alimentos regionais podem ser utilizados para a elaboração de preparações saborosas. É bem reconhecido que as oficinas culinárias são formas lúdicas, dinâmicas e prazerosas de aprendizado, como estratégias efetivas para a promoção de hábitos alimentares saudáveis ${ }^{19,20}$.

$\mathrm{O}$ valor obtido no teste de aceitabilidade do lanche oferecido aos alunos foi considerado excelente na Escola A (95,8\%); e na Escola B (84\%) alcançou boa aceitação, conforme o FNDE $n^{\circ} 26 / 2013^{9}$, o que se assemelhou ao resultado do estudo de Raphaelli et al. ${ }^{21}$ em escolas de zona rural, com excelente aceitação de lanches em detrimento das refeições. Por outro lado, estudando a aceitabilidade de crianças e 
adolescentes, Garcia et al. ${ }^{22}$, verificaram maior aceitação de lanches por adolescentes, enquanto as crianças expressaram maior aceitação pelas refeições.

Esses resultados indicam necessidade de ações de educação alimentar e nutricional para o público escolar, pois o hábito alimentar e a presença de cantinas escolares terceirizadas influenciam na aceitação da alimentação escolar, com impacto direto no estado nutricional da criança ${ }^{23,24}$.

Os hábitos alimentares de crianças e adolescentes estão se caracterizando pela substituição de alimentos tradicionais saudáveis por outros de baixo valor nutricional, elevada concentração de energia e de fácil consumo, que não fazem ou não devem fazer parte dos cardápios escolares ${ }^{25}$. Trabalhar metodologias combinadas, com o intuito de promover o consumo alimentar saudável, é uma estratégia no ambiente escolar, pois contribui para a aquisição de novos conhecimentos e vivências que melhoram a qualidade de vida dos estudantes e promovem a sustentabilidade ${ }^{26}$. Os resultados deste estudo foram satisfatórios e devem ser continuados envolvendo mais atores sociais, como a família e outros integrantes da escola; e, como propõem Prado et al. ${ }^{1}$, englobar o ambiente e a produção de alimentos, pautada na sustentabilidade.

\section{Conclusão}

As metodologias ativas aplicadas no desenvolvimento de EAN demonstraram ser estratégias eficazes no processo de aquisição do conhecimento a respeito do que são alimentos regionais, alimentação saudável e valorização da cultura alimentar local, pois obtiveram-se ótimos resultados em sua utilização com o público escolar infantil neste estudo.

O emprego dessas metodologias na educação em saúde despertou a atenção e o interesse dos escolares. Assim, sugere-se que parcerias sejam firmadas com as instituições de ensino superior de forma que o conhecimento possa ser compartilhado e que essas atividades possam ser utilizadas no processo de ensino-aprendizagem com maior frequência, em particular, para melhor orientar as escolhas alimentares dos estudantes. Caso os docentes do ensino fundamental não estejam adequadamente capacitados, a parceria entre as instituições de ensino superior, as escolas públicas e a comunidade pode aprimorar e consolidar o conhecimento, tornando mais efetivo o processo de ensino-aprendizagem com os temas saúde e alimentação do escolar.

\section{Agradecimentos}

À Pró-Reitoria de Extensão da Universidade Federal do Pará, pelo financiamento da bolsista do projeto "Cultivando Hortas Escolares como Eixo Gerador de Segurança Alimentar, Nutricional e Sustentabilidade", que possibilitou a realização da atividade nas escolas públicas, às quais estendemos o nosso agradecimento, à Direção, aos docentes, aos alunos sujeitos da pesquisa e aos voluntários do projeto.

\section{Referências}

1. Prado BG, Fortes ENS, Lopes MAL, Guimarães LV. Ações de educação alimentar e nutricional para escolares: um relato de experiência. Demetra Alim Nut Saúde. 2016;11(2):369-82. http://dx.doi.org/10.12957/demetra.2016.16168.

2. Sociedade Brasileira de Pediatria (SBP). Manual de orientação para a alimentação do lactente, do pré-escolar, do escolar, do adolescente e na escola. Rio de Janeiro: Departamento de Nutrologia; 2012.

3. Brasil. Ministério da Saúde. Alimentos regionais brasileiros. 2a ed. Brasília: Ministério da Saúde; 2015.

4. Martins MC, Ferreira AMV, Nascimento LA, Aires JS, Almeida PC, Ximenes LB. Influência de uma estratégia educativa na promoção do uso de alimentos regionais. Rev Rene. 2015;16(2):242-9.

5. Oliveira ACS, Souza LMB. Avaliação da frequência do consumo de alimentos ultraprocessados de crianças menores de 10 anos. SADSJ - South Am Dev Soc J. 2017;2(6):143-154.

6. Brasil. Ministério do Desenvolvimento Social e Combate à Fome. Marco de referência de educação alimentar e nutricional para as políticas públicas. Brasília: Ministério do Desenvolvimento e Combate à Fome; 2012.

7. Maia PA, Martin ALAR, Cristo CS. Aprendizagem Ativa na Educação em Saúde: Percurso Histórico e Aplicações. Rev Bras Educ Med. 2015;39(1):143-58. http://dx.doi.org/10.1590/1981-52712015v39n1e00602014. 

do aprendizado [dissertação de mestrado]. Lorena: Universidade de São Paulo - USP; 2017.

9. Brasil. Ministério da Educação. Fundo Nacional de Desenvolvimento da Educação. Resolução n. 26 , de 17 de junho de 2013. Atendimento da alimentação escolar aos alunos da educação básica no âmbito do Programa Nacional de Alimentação Escolar - PNAE. Diário Oficial da União, Brasília, DF, 18 jun. 2013. Seção 1.

10. Brasil. Ministério da Educação. Manual para aplicação dos testes de aceitabilidade no Programa Nacional de Alimentação Escolar (PNAE). Brasília: Ministério da Educação; 2010.

11. Brasil. Ministério da Educação. Manual para aplicação dos testes de aceitabilidade no Programa Nacional de Alimentação Escolar (PNAE). Brasília: Ministério da Educação; 2010.

12. Ayres M, Ayres J, Ayres M, et al. BioEstat 5.3: Aplicações estatísticas nas áreas das ciências biológicas e médicas. 5a ed. Belém: CNPq; 2007.

13. Filgueiras MC, Lima NVR, Souza SS, Moreira AKF. Prevalência de obesidade em crianças de escolas públicas. Rev Ciên Saúde. 2012;5(1):41-7. http://dx.doi.org/10.15448/1983-652X.2012.1.10257.

14. Silva TKR, Camargo GA, Nunes CEC, Silva CC, Ballard CR. Avaliação da alimentação escolar e do estado nutricional de adolescentes. Rev Aten Saúde. 2017;15(51):62-8. http://dx.doi.org/10.13037/ras.vol15n51.4278.

15. Luchetti AJ, Moreale VC, Parro MC. Educação em saúde: uma experiência com teatro de fantoches no ensino nutricional de escolares. Rev CuidArte Enf. 2011;5(2):97-103.

16. Aringhieri LFA, Silva FAR. Teatro de fantoches: uma apresentação lúdica de física moderna em escolas do ensino fundamental. Scien Plena. 2017;13(1):1-10. http://dx.doi.org/10.14808/10.14808/sci.plena.2017.012705.

17. Vasconcelos MIO, Farias QLT, Nascimento FGN, Cavalcante ASP, Mira QLM, Queiroz MVO. Educação em saúde na atenção básica: uma análise das ações com hipertensos. Rev APS. 2017;20(2):253-62.

18. Dias ESM, Rodrigues ILA, Miranda HR, Corrêa JA. Roda de conversa como estratégia de educação em saúde para a enfermagem. J Res: fundam Care. 2018;10(2):379-384.

19. Ferreira FV, Azevedo FM, Teixeira BA, Silva LSB, Morais JR, Novaes JF. Oficina culinária como estratégia de educação alimentar e nutricional na comunidade Córrego São João e setores, Viçosa, Minas Gerais: uma parceria entre o Programa de Educação Tutorial em Nutrição/Universidade Federal de Viçosa e a Empresa de Assistência Técnica e Extensão Rural. J Manag Prim Health Care. 2016;7(1):75-75.

20. Almeida JC, Mendonça AAF, Gonçalves IE, Batista LM. Intervenção nutricional através de oficinas culinárias e palestras educativas: aplicativas e influência no estado nutricional de adultos. RBONE. 2018;12(69):126-31.

21. Raphaelli CO, Passos LDF, Couto SF, Helbig E, Madruga SW. Adesão e aceitabilidade de cardápios da alimentação escolar do ensino fundamental de escolas de zona rural. Braz J Food Technol. 2017;20(e2016112):1-9. http://dx.doi. org/10.1590/1981-6723.11216.

22. Garcia LS, Rocha TC, Araújo ND, Viana KS, Maranhão HC. Aceitabilidade da Alimentação Escolar por crianças e adolescentes de um município rural do Nordeste brasileiro. Rev Catussaba. 2016;5(1):85-94.

23. Sacchetto KK, Madaschi V, Barbosa GHL, et al. O ambiente lúdico como fator motivacional na aprendizagem escolar. Rev Cad Pós-Grad Distúrb Desenvolv. 2011;11(1):28-36.

24. Cesar JT, Valentim EA, Almeida CCB, Schieferdecker MEM, Schmidt ST. Alimentação escolar no Brasil e Estados Unidos: uma revisão integrativa. Rev Cienc Saúde Coletiva. 2018;23(4):991-1007. http://dx.doi.org/10.1590/1413-81232018233.01582016.

25. Barbosa VCB Fo, Campos W, Lopes AS. Epidemiology of physical inactivity, sedentary behaviors, and unhealthy eating habits among Brazilian adolescents: a systematic review. Cien Saude Colet. 2014;19(1):173-93. http://dx.doi.org/10.1590/141381232014191.0446. PMid:24473615.

26. Triches RM. Promoção do consumo alimentar sustentável no contexto da alimentação escolar. Trab Educ Saúde. 2015;13(3):75771. http://dx.doi.org/10.1590/1981-7746-sip00061.

\section{Contribuição dos autores}

Adriene Carvalho da Conceição - participou da concepção e desenvolvimento, da supervisão, da coleta, tratamento e análise dos dados e da redação e revisão do manuscrito. Bárbara Valéria de Souza Santos - participou da coleta, tratamento e análise dos dados, levantamento da literatura, da redação e revisão do manuscrito. Elaine do Socorro Brabo Duarte - participou do levantamento da literatura e da redação do manuscrito. Elen Dayane Albuquerque Cardoso - participou da concepção e desenvolvimento, da supervisão, da coleta, tratamento e análise dos dados e da redação e revisão do manuscrito. Erica Costa da Costa - participou da concepção e desenvolvimento, da supervisão, da coleta, tratamento e análise dos dados e da redação e revisão do manuscrito. Leandro Orlando Sousa Da Silva, participou da elaboração do desenho metodológico, da execução do projeto, da coleta e da interpretação dos dados. Reia Sílvia Lemos da Costa e Silva Gomes - participou do levantamento da literatura, da redação e da revisão crítica do manuscrito. Xaene Maria Fernandes Duarte Mendonça. - participou da Concepção e desenvolvimento da pesquisa, da coleta e da interpretação dos dados, do levantamento bibliográfico, da redação e da revisão da manuscrito. 